\title{
ORDER ISOMORPHISMS ON FUNCTION SPACES
}

\author{
DENNY H. LEUNG AND LEI LI
}

\begin{abstract}
The classical theorems of Banach and Stone 3, 15, Gelfand and Kolmogorov [7] and Kaplansky [14] show that a compact Hausdorff space $X$ is uniquely determined by the linear isometric structure, the algebraic structure, and the lattice structure, respectively, of the space $C(X)$. In this paper, it is shown that for rather general subspaces $A(X)$ and $A(Y)$ of $C(X)$ and $C(Y)$ respectively, any linear bijection $T: A(X) \rightarrow A(Y)$ such that $f \geq 0$ if and only if $T f \geq 0$ gives rise to a homeomorphism $h: X \rightarrow Y$ with which $T$ can be represented as a weighted composition operator. The three classical results mentioned above can be derived as corollaries. Generalizations to noncompact spaces and other function spaces such as spaces of uniformly continuous functions, Lipschitz functions and differentiable functions are presented.
\end{abstract}

\section{INTRODUCTION}

A well established area of research seeks to determine the relationship between the structure of a set and the properties of certain function spaces defined on it. A closely related question is to determine the general form of an operator mapping between various function spaces. In the case of spaces of continuous functions, a vast literature exists. A good summary of the classical results is the text of Gillman and Jerison [10]. From the classical period, three results in this area stand out; namely the theorems of Banach and Stone, Gelfand-Kolmogorov and Kaplansky (see Corollary 8 below). When $X$ and $Y$ are compact Hausdorff spaces, these results determine the precise forms of the norm isometries, algebra isomorphisms and vector lattice isomorphisms between $C(X)$ and $C(Y)$ respectively. In particular, the existence of any one of these three types of operators lead to homeomorphism between $X$ and $Y$. More recent results of Banach-Stone type are found in [1, 2, 8, for example.

Kadison [13] showed that a linear order isomorphism $T$ between two $C^{*}$ algebras which maps the identity to the identity is a $C^{*}$-isomorphism, i.e., $T$ satisfies $T\left(x^{2}\right)=(T x)^{2}$ for all $x$. In the commutative case, it follows that for any compact Hausdorff spaces $X$ and $Y$, any linear order isomorphism from $C(X)$ onto $C(Y)$ that maps the constant function 1 to the constant function 1 is an algebra isomorphism. The theorem of Gelfand-Kolmogorov then implies that $T$ is a composition operator. Order isomorphisms, even

Research of the first author was partially supported by AcRF project no. R-146-000157-112. 
nonlinear ones, have been studied by various authors, see, e.g., [4, 5, 6, 8, 9, 11, 12]. These results concern order isomorphisms between specific function spaces. Moreover, the spaces considered are all either lattices or algebras of functions. One of the aims of the present paper is to provide a unified treatment of linear order isomorphisms within a general framework. In particular, our results apply to all unital function lattices that separate points from closed sets and many function algebras.

For quite general subspaces $A(X)$ and $A(Y)$ of $C(X)$ and $C(Y)$ respectively, where $X$ and $Y$ are compact Hausdorff spaces, Theorem 1 in 92 determines the precise form of a linear order isomorphism $T: A(X) \rightarrow A(Y)$ and shows that the existence of such a map leads to homeomorphism of $X$ and $Y$. The classical results cited above can all be subsumed under this theorem. We also provide an example of a space to which the theorem applies which is neither a lattice nor an algebra.

In \$3, we apply a Stone-Čech like compactification procedure to extend Theorem 1 to noncompact spaces. As a result, existence of a linear order isomorphism $T: A(X) \rightarrow A(Y)$ gives rise to a homeomorphism between some compactifications of $X$ and $Y$ respectively. In 4 , we show that under certain circumstances, the homeomorphism obtained restricts to a homeomorphism between $X$ and $Y$.

Because much of the paper is concerned with maps preserving order, we consider only real vector spaces.

The authors wish to thank the referee whose astute comments on the first submission of this paper prompted much further reflection and great improvements in both the content and exposition of the paper.

\section{ORDER ISOMORPHISMS OF SPACES OF CONTINUOUS FUNCTIONS ON COMPACT HAUSDORFF SPACES}

Let $X$ be a topological space and let $A(X)$ be a vector subspace of $C(X)$. $A(X)$ is said to separate points from closed sets if given $x \in X$ and a closed set $F$ in $X$ not containing $x$, there exists $f \in A(X)$ such that $f(x)=1$ and $f(F) \subseteq\{0\}$. If, in addition, $f$ can be chosen to have values in $[0,1]$, then we say that $A(X)$ precisely separates points from closed sets. It is clear that any sublattice of $C(X)$ that separates points from closed sets does so precisely. Let $Y$ be a topological space and let $A(Y)$ be a vector subspace of $C(Y)$, a linear bijection $T: A(X) \rightarrow A(Y)$ is an order isomorphism if $f \geq 0$ if and only if $T f \geq 0$. The aim of this section is to prove the following theorem.

Theorem 1. Let $X, Y$ be compact Hausdorff spaces and let $A(X)$ and $A(Y)$ be subspaces of $C(X)$ and $C(Y)$ respectively that contain the constant functions and precisely separate points from closed sets. If $T: A(X) \rightarrow A(Y)$ is a linear order isomorphism, then there is a homeomorphism $h: X \rightarrow Y$ such that $T f=T 1_{X} \cdot f \circ h^{-1}$ for all $f \in A(X)$.

The proof is divided into a number of steps listed below, from Proposition 2 to Proposition [6. If $f \in A(X)$ or $A(Y)$, let $Z(f)=\{f=0\}$. 
Proposition 2. For any $x_{0} \in X$, let

$$
\mathcal{Z}_{x_{0}}=\left\{Z(T f): f \in A(X), f \geq 0, f\left(x_{0}\right)=0\right\} .
$$

Then $\mathcal{Z}_{x_{0}}$ has the finite intersection property.

Proof. Suppose that $f_{i} \in A(X), f_{i} \geq 0$, and $f_{i}\left(x_{0}\right)=0,1 \leq i \leq n$. Let $f=\sum_{i=1}^{n} f_{i}$. Then $f \in A(X), f \geq 0$, and $f\left(x_{0}\right)=0$. In particular, $T f \geq 0$. If $Z(T f)=\emptyset$, that is, $(T f)(y)>0$ for all $y \in Y$, there exists $\varepsilon>0$ such that $T f-\varepsilon T 1_{X} \geq 0$. Thus $f-\varepsilon 1_{X} \geq 0$, which is manifestly untrue. Let $y_{0} \in Y$ be such that $T f\left(y_{0}\right)=0$. Then $\sum_{i=1}^{n} T f_{i}\left(y_{0}\right)=0$. Since $T f_{i} \geq 0$, $T f_{i}\left(y_{0}\right)=0,1 \leq i \leq n$. Thus $y_{0} \in \cap_{i=1}^{n} Z\left(T f_{i}\right)$.

Define $\mathcal{Z}_{y_{0}}$ similarly for $y_{0} \in Y$, using the operator $T^{-1}$ in place of $T$. By Proposition 2. $\cap \mathcal{Z}_{x_{0}}$ and $\cap \mathcal{Z}_{y_{0}}$ are nonempty for all $x_{0} \in X, y_{0} \in Y$.

Proposition 3. Let $x_{0} \in X$ and $y_{0} \in Y$. Then $y_{0} \in \cap \mathcal{Z}_{x_{0}}$ if and only if $x_{0} \in \cap \mathcal{Z}_{y_{0}}$.

Proof. Suppose that $y_{0} \in \cap \mathcal{Z}_{x_{0}}$ but $x_{0} \notin \cap \mathcal{Z}_{y_{0}}$. Choose $x_{1} \in \cap \mathcal{Z}_{y_{0}}$. Then $x_{1} \neq x_{0}$. There exists $f \in A(X)$, range $f \subseteq[0,1]$, such that $f\left(x_{1}\right)=1$ and $f\left(x_{0}\right)=0$. Since $y_{0} \in \cap \mathcal{Z}_{x_{0}}, T f\left(y_{0}\right)=0$. As $T$ is an order isomorphism, $T f \geq 0$. Then $x_{1} \in \cap \mathcal{Z}_{y_{0}}$ implies that $f\left(x_{1}\right)=T^{-1}(T f)\left(x_{1}\right)=0$, contrary to the choice of $f$. The "if" part of the proposition follows by symmetry.

Proposition 4. $\cap \mathcal{Z}_{x_{0}}$ contains exactly one point.

Proof. It has already been observed that $\cap \mathcal{Z}_{x_{0}}$ is nonempty. Suppose that there are distinct points $y_{1}$ and $y_{2}$ in $\cap \mathcal{Z}_{x_{0}}$. Choose $g \in A(Y)$ such that range $g \subseteq[0,1], g\left(y_{1}\right)=0$ and $g\left(y_{2}\right)=1$. By Proposition 3, $x_{0} \in \cap \mathcal{Z}_{y_{1}}$. Hence $T^{-1} g\left(x_{0}\right)=0$. Since $y_{2} \in \cap \mathcal{Z}_{x_{0}}, T\left(T^{-1} g\right)\left(y_{2}\right)=0$. Thus $g\left(y_{2}\right)=0$, yielding a contradiction.

Proposition 5. $T 1_{X}(y)>0$ and $T^{-1} 1_{Y}(x)>0$ for all $x \in X$ and all $y \in Y$.

Proof. Suppose that there exists $y_{0} \in Y$ such that $T 1_{X}\left(y_{0}\right)=0$. For any $f \in A(X)$, there exists $0 \leq c \in \mathbb{R}$ such that $-c 1_{X} \leq f \leq c 1_{X}$. Then $-c T 1_{X}\left(y_{0}\right) \leq T f\left(y_{0}\right) \leq c T 1_{X}\left(y_{0}\right)$ for all $f \in A(X)$. Hence $T f\left(y_{0}\right)=0$ for all $f \in A(X)$. This is a contradiction since $T$ maps onto $A(Y)$ and $A(Y)$ contains all constant functions.

Define $h: X \rightarrow Y$ by $h\left(x_{0}\right)=y_{0}$, where $\left\{y_{0}\right\}=\cap \mathcal{Z}_{x_{0}}$.

Proposition 6. $h$ is a homeomorphism from $X$ onto $Y$ so that $T f=T 1_{X}$. $f \circ h^{-1}$ for all $f \in A(X)$ and all $y \in Y$.

Proof. The injectivity of $h$ follows from Proposition 3. If $y \in Y$, let $\{x\}=$ $\cap \mathcal{Z}_{y}$. By Proposition 3, $y \in \cap \mathcal{Z}_{x}$. Thus $h(x)=y$. This shows that $h$ is surjective.

Suppose that $x_{0} \in X$ and $y_{0}=h\left(x_{0}\right)$. Let $f \in A(X)$ and let $m=$ $\min \{f(x): x \in X\}$. Given $\varepsilon>0$, let $U$ be an open neighborhood of $x_{0}$ 
so that $f(x)>f\left(x_{0}\right)-\varepsilon$ for all $x \in U$. There exists $g_{1} \in A(X)$ such that range $g_{1} \subseteq[0,1], g_{1}\left(x_{0}\right)=1$ and $g_{1}(x)=0$ for all $x \notin U$. Since $1_{X}-g_{1} \geq 0,\left(1_{X}-g_{1}\right)\left(x_{0}\right)=0$ and $y_{0} \in \cap \mathcal{Z}_{x_{0}}, T\left(1_{X}-g_{1}\right)\left(y_{0}\right)=0$. Hence $\left(T g_{1}\right)\left(y_{0}\right)=\left(T 1_{X}\right)\left(y_{0}\right)$. Now

$$
f-m 1_{X}-\left(f\left(x_{0}\right)-m-\varepsilon\right) g_{1} \geq 0 .
$$

Thus

$$
(T f)\left(y_{0}\right) \geq m\left(T 1_{X}\right)\left(y_{0}\right)+\left(f\left(x_{0}\right)-m-\varepsilon\right)\left(T g_{1}\right)\left(y_{0}\right),
$$

that is, $(T f)\left(y_{0}\right) \geq\left(T 1_{X}\right)\left(y_{0}\right)\left(f\left(x_{0}\right)-\varepsilon\right)$. As $\varepsilon>0$ is arbitrary, $T f\left(y_{0}\right) \geq$ $\left(T 1_{X}\right)\left(y_{0}\right) f\left(x_{0}\right)$. Applying the argument to $-f$ yields the reverse inequality. Thus $T f\left(y_{0}\right)=\left(T 1_{X}\right)\left(y_{0}\right) f\left(x_{0}\right)$.

It remains to show that $h$ is a homeomorphism. Let $x_{0} \in X$ and $y_{0}=$ $h\left(x_{0}\right)$. Suppose that $V$ is an open neighborhood of $y_{0}$ in $Y$. There exists $g \in A(Y)$ such that $g\left(y_{0}\right)=1$, range $g \subseteq[0,1]$, and $g=0$ outside $V$. Since $g \geq 0, T^{-1} g \geq 0$. If $T^{-1} g\left(x_{0}\right)=0$, then $y_{0} \in Z\left(T\left(T^{-1} g\right)\right)=Z(g)$, contrary to the choice of $g$. Thus $T^{-1} g\left(x_{0}\right)>0$. Therefore, the set $U=\left\{T^{-1} g>0\right\}$ is an open neighborhood of $x_{0} \in X$. Suppose that $x \in U$. By the previous paragraph,

$$
g(h(x))=T\left(T^{-1} g\right)(h(x))=\left(T 1_{X}\right)(h(x)) T^{-1} g(x)>0 .
$$

Hence $h(x) \in V$. This proves that $h$ is continuous. Since $h$ is a continuous bijection between compact Hausdorff spaces, it is a homeomorphism.

Remark. Applying Theorem 1 to the map $T^{-1}$ gives a homeomorphism $k: Y \rightarrow X$ such that $T^{-1} g=T^{-1} 1_{Y} \cdot g \circ k^{-1}$. Because of Proposition 3, $k$ must be $h^{-1}$.

The classical theorems of Banach [3] and Stone [15], Gelfand and Kolmogorov [7] and Kaplansky [14] show that a compact Hausdorff space $X$ is uniquely determined by the linear isometric structure, the algebraic structure, and the lattice structure, respectively, of the space $C(X)$. These results can be subsumed under Theorem 1. As usual, $C(X)$ and $C(Y)$ are endowed with their respective supremum norms.

Lemma 7. Let $X$ and $Y$ be compact Hausdorff spaces. If $T: C(X) \rightarrow C(Y)$ is an onto linear isometry, then $\left|T 1_{X}\right|=1_{Y}$ and, for any $f \in C(X), f \geq 0$ if and only if $T f \cdot T 1_{X} \geq 0$.

Proof. Since $T$ is an isometry, $\left\|T 1_{X}\right\|=1$. Suppose that there exists $y_{0} \in Y$ such that $\left|T 1_{X}\left(y_{0}\right)\right|<1$. There exists a neighborhood $V$ of $y_{0}$ and $a>0$ such that $\left|T 1_{X}(y)\right|<1-a$ for all $y \in V$. Choose $g \in C(Y)$ such that $g\left(y_{0}\right)=1$, range $g \subseteq[0,1]$ and $g=0$ outside $V$. Then $\left\|1_{X}+a T^{-1} g\right\|=\left\|T 1_{X}+a g\right\| \leq 1$. Thus $1+a T^{-1} g(x) \leq 1$ for all $x \in X$. Therefore, $T^{-1} g \leq 0$. As $\left\|T^{-1} g\right\|=$ $\|g\|=1$, there must be some $x_{0} \in X$ where $T^{-1} g\left(x_{0}\right)=-1$. Then

$$
2=\left|\left(1_{X}-T^{-1} g\right)\left(x_{0}\right)\right| \leq\left\|1_{X}-T^{-1} g\right\| \leq\left\|1_{X}\right\|+\left\|T^{-1} g\right\|=2 .
$$


Hence $\left\|T 1_{X}-g\right\|=\left\|1_{X}-T^{-1} g\right\|=2$. However, if $y \in V$, then

$$
\left|\left(T 1_{X}-g\right)(y)\right| \leq\left|T 1_{X}(y)\right|+\|g\|<1-a+1<2 .
$$

On the other hand, if $y \notin V$, then $\left|\left(T 1_{X}-g\right)(y)\right|=\left|T 1_{X}(y)\right| \leq\left\|1_{X}\right\|=1$. This proves that $\left\|T 1_{X}-g\right\|<2$, contrary to the above. Therefore, $\left|T 1_{X}\right|=$ $1_{Y}$.

Given $f \in C(X), f \geq 0$ if and only if $\|f-\| f\left\|1_{X}\right\| \leq\|f\|$. Since $T$ is an isometry, this is equivalent to $\|T f-\| T f\left\|T 1_{X}\right\| \leq\|T f\|$. By the above, $\left|T 1_{X}\right|=1_{Y}$. Thus the final inequality holds if and only if $T f \cdot T 1_{X} \geq 0$.

A linear bijection $T: C(X) \rightarrow C(Y)$ is

(a) a lattice isomorphism if $|T f|=T|f|$ for all $f \in C(X)$;

(b) an algebra isomorphism if $T 1_{X}=1_{Y}$ and $T(f g)=T f \cdot T g$ for all $f, g \in C(X)$.

Corollary 8. Let $X$ and $Y$ be compact Hausdorff spaces and let $T: C(X) \rightarrow$ $C(Y)$ be a linear bijection.

(a) (Banach-Stone) If $T$ is an isometry, then there is a homeomorphism $h: X \rightarrow Y$ and a function $g \in C(Y),|g|=1_{Y}$, such that $T f=$ $g \cdot f \circ h^{-1}$ for all $f \in C(X)$.

(b) (Kaplansky) If $T$ is a lattice isomorphism, then there is a homeomorphism $h: X \rightarrow Y$ and a function $g \in C(Y), g(y)>0$ for all $y \in Y$, such that $T f=g \cdot f \circ h^{-1}$ for all $f \in C(X)$.

(c) (Gelfand and Kolmogorov) If $T$ is an algebra isomorphism, then there is a homeomorphism $h: X \rightarrow Y$ such that $T f=f \circ h^{-1}$ for all $f \in C(X)$.

Proof. Let $g=T 1_{X}$. For case (a), it follows from Lemma 7 that $|g|=1_{Y}$ and that the operator $\widetilde{T}: C(X) \rightarrow C(Y)$ given by $\widetilde{T}(f)=T f / g$ is a linear order isomorphism. By Theorem 1, there exists a homeomorphism $h: X \rightarrow Y$ such that $\widetilde{T} f=\widetilde{T} 1_{X} \cdot f \circ h^{-1}$. It follows easily that $T f=g \cdot f \circ h^{-1}$. For cases (b) and (c), it is clear that $T$ is a linear order isomorphism. Proposition 5 gives that $g(y)>0$ for all $y \in Y$. Moreover, $g=1_{Y}$ for case (c). The representation of $T$ follows immediately from Theorem 11.

Remark. Lemma 7 may be extended to linear isometries $T: C(X, \mathbb{C}) \rightarrow$ $C(Y, \mathbb{C})$. As a result, Corollary 8 (a) for complex isometries may also be derived in a similar manner.

Example. There is a subspace $\mathcal{X}$ of $C[0,1]$ that contains constants and precisely separates points from closed sets, so that $\mathcal{X}$ is neither a sublattice nor a subalgebra of $C[0,1]$.

Define $\theta: \mathbb{R} \rightarrow \mathbb{R}$ by $\theta(t)=\sin \frac{\pi t}{2}$ and $g: \mathbb{R} \rightarrow \mathbb{R}$ by $g(t)=0, \theta(t), 1$ reespectively if $t \leq 0,0<t<1, t \geq 1$ respectively. Denote by $t$ the identity function $t \mapsto t$ on $[0,1]$ or $\mathbb{R}$, as the case may be. If $\mathcal{F}$ is a set of real-valued functions defined on $[0,1]$ or $\mathbb{R}$, let $g \circ \mathcal{F}=\{g \circ f: f \in \mathcal{F}\}$. Denote by $\mathcal{X}_{1}$ and $\Sigma_{1}$ respectively the span of the functions 1 and $t$ in $C[0,1]$ and $C(\mathbb{R})$ 
respectively. Let $\mathcal{X}_{n+1}=\operatorname{span}\left\{\mathcal{X}_{n} \cup g \circ \mathcal{X}_{n}\right\}$ and $\Sigma_{n+1}=\operatorname{span}\left\{\Sigma_{n} \cup \theta \circ \Sigma_{n}\right\}$. Then set $\mathcal{X}=\cup_{n} \mathcal{X}_{n}$ and $\Sigma=\cup_{n} \Sigma_{n}$. It is easy to see that $\mathcal{X}$ is a subspace of $C[0,1]$ and that $\Sigma$ is a subspace of $C(\mathbb{R})$. Moreover, $\Sigma$ consists of real analytic functions on $\mathbb{R}$. We claim that $\mathcal{X}$ has the desired properties.

First observe that for any $a<b$ in $[0,1]$, the linear function $f$ on $[0,1]$ such that $f(a)=0$ and $f(b)=1$ belongs to $\mathcal{X}_{1}$. Hence $g \circ f \in \mathcal{X}_{2} \subseteq \mathcal{X} . g \circ f$ has the property that $g \circ f(t)=0$ if $t \leq a, 1$ if $t \geq b$ and $0<g \circ f(t)<1$ if $a<t<b$. Taking differences of two such functions shows that $\mathcal{X}$ separates points from closed sets in $[0,1]$. By construction, $g \circ f \in \mathcal{X}$ for any $f \in \mathcal{X}$. Thus $\mathcal{X}$ satisfies conditions (a) and (b) of Definition 11 below. Since $\mathcal{X}$ consists of bounded functions, condition (c) is also satisfied. It follows from Lemma 13 that $\mathcal{X}$ precisely separates points form closed sets in $[0,1]$.

Lemma 9. If $f \in \mathcal{X}_{n}$ and $I$ is a nondegenerate interval in $[0,1]$, then there is a nondegenerate interval $J \subseteq I$ and a function $u \in \Sigma_{n}$ such that $f=u$ on $J$.

Proof. Induct on $n$. The case $n=1$ is trivial. Assume that the result holds for some $n$ and let $f \in \mathcal{X}_{n+1}$. Then $f=f_{0}+\sum_{i=1}^{m} c_{i} g \circ f_{i}$, where $f_{i} \in \mathcal{X}_{n}, 0 \leq$ $i \leq m$. By the inductive assumption, there exist a nondegenerate interval $I_{0} \subseteq I$ and $u_{0} \in \Sigma_{n}$ such that $f_{0}=u_{0}$ on $I_{0}$. If $f_{1}\left(I_{0}\right) \cap(0,1)=\emptyset$, then $g \circ f_{1}\left(I_{0}\right) \subseteq\{0,1\}$. By connectedness of $I_{0}$ and continuity of $g \circ f_{1}, g \circ f_{1}$ takes constant value, say $c_{1}(=0$ or 1$)$ on $I_{0}$. In this case, let $I_{1}=I_{0}$ and $u_{1}=c_{1}$. Then $g \circ f_{1}=c_{1}=\theta \circ u_{1}$ on $I_{1}$. Otherwise, $f_{1}\left(I_{0}\right) \cap(0,1) \neq \emptyset$. There is a nondegenerate interval $I_{0}^{\prime} \subseteq I_{0}$ such that $f_{1}\left(I_{0}^{\prime}\right) \subseteq(0,1)$. Then $g \circ f_{1}=\theta \circ f_{1}$ on $I_{0}^{\prime}$. By the inductive assumption, there exist a nondegenerate interval $I_{1} \subseteq I_{0}^{\prime}$ and $u_{1} \in \Sigma_{n}$ such that $f_{1}=u_{1}$ on $I_{1}$. Then $g \circ f_{1}=\theta \circ f_{1}=\theta \circ u_{1}$ on $I_{1}$. Continue to choose nondegenerate intervals $I_{0} \supseteq I_{1} \supseteq \cdots \supseteq I_{m}$, $u_{0}, \ldots, u_{m} \in \Sigma_{n}$ such that $g \circ f_{i}=\theta \circ u_{i}$ on $I_{i}, 1 \leq i \leq m$, and $f_{0}=u_{0}$ on $I_{0}$. Then $f=u_{0}+\sum_{i=1}^{m} c_{i} \theta \circ u_{i}$ on $I_{m}$ and the latter function belongs to $\Sigma_{n+1}$.

Lemma 10. If $f$ is a real analytic function on $\mathbb{R}$ and $f_{\mid[0,1]} \in \mathcal{X}$, then $f \in \Sigma$.

Proof. Let $f_{0}=f_{\mid[0,1]}$. By Lemma 9, there is a nondegenerate interval $J$ and $u \in \Sigma$ such that $f_{0}=u$ on $J$. Since both $f$ and $u$ are real analytic on $\mathbb{R}, f=u$ on $\mathbb{R}$.

We can now verify the remaining properties of $\mathcal{X}$ stated above, namely that $\mathcal{X}$ is neither a sublattice nor a subalgebra of $C[0,1]$. If $\mathcal{X}$ is an algebra, then $t^{2} \in \mathcal{X}$. By Lemma 10, $t^{2}$ (as a function on $\mathbb{R}$ ) belongs to $\Sigma$. We show that this is impossible by showing that $\lim _{t \rightarrow \infty} f(t) / t^{2}=0$ for any $f \in \Sigma$. Indeed, the statement holds for any $f \in \Sigma_{1}$. Inductively, any $f \in \Sigma_{n+1}$ can be written as $f=f_{0}+\sum_{i=1}^{m} c_{i} \theta \circ f_{i}$, where $f_{i} \in \mathcal{X}_{n}, 0 \leq i \leq m$. By induction, $\lim _{t \rightarrow \infty} f_{0}(t) / t^{2}=0$. Since $0 \leq \theta \circ f_{i} \leq 1$, the induction is complete.

Finally, suppose that $\mathcal{X}$ is a lattice. Then $t \wedge \frac{1}{2} \in \mathcal{X}$. Say it belongs to $\mathcal{X}_{n}$. It is easy to check via induction that

$$
\mathcal{X}_{n}=\mathcal{X}_{1}+\operatorname{span}\left(g \circ \mathcal{X}_{1}\right)+\cdots+\operatorname{span}\left(g \circ \mathcal{X}_{n-1}\right) .
$$


Then we can write $f=f_{0}+g \circ f_{1}+\cdots+g \circ f_{n-1}$, where $f_{0} \in \mathcal{X}_{1}, f_{i} \in \mathcal{X}_{i}$, $1 \leq i \leq n-1$. Say $f_{0}=a+b t$. By Lemma 9, there exist nondegenerate intervals $J_{1} \subseteq[0,1 / 2], J_{2} \subseteq[1 / 2,1]$, functions $u_{i}, v_{i} \in \Sigma, 1 \leq i \leq n-1$, such that

$$
f= \begin{cases}a+b t+\sum_{i=1}^{n-1} \theta \circ u_{i} & \text { on } J_{1} \\ a+b t+\sum_{i=1}^{n-1} \theta \circ v_{i} & \text { on } J_{2} .\end{cases}
$$

Then $t=a+b t+h_{1}$ on $J_{1}$ and $1 / 2=a+b t+h_{2}$ on $J_{2}$, where $h_{1}$ and $h_{2}$ are bounded real analytic functions. Thus these equations hold on $\mathbb{R}$. Dividing both equations by $t$ and taking limits as $t \rightarrow \infty$ gives $1=b$ and $0=b$, which is absurd.

\section{ORder isomorphisms on COMPLETELY REGULAR SPACES}

In this section, we employ the method of compactification to extend results in the previous section to more general function spaces. Let $X$ be a (Hausdorff) completely regular space and let $A(X)$ be a subspace of $C(X)$ that separates points from closed sets. Denote by $\mathbb{R}_{\infty}$ the interval $[-\infty, \infty]$ with the order topology. The map $i: X \rightarrow \mathbb{R}_{\infty}^{A(X)}, i(x)(\varphi)=\varphi(x)$, is a

homeomorphic embedding. Let $\mathcal{A} X$ be the closure of $i(X)$ in $\mathbb{R}_{\infty}^{A(X)}$. Then $\mathcal{A} X$ is a compact Hausdorff space. Identify $X$ with $i(X)$ and regard $X$ as a subspace of $\mathcal{A} X$. For each $f \in A(X)$, there is a unique continuous extension $\hat{f}: \mathcal{A} X \rightarrow \mathbb{R}_{\infty}$ given by $\hat{f}(x)=x(f)$. When $A(X)=C(X), \mathcal{A} X$ coincides with the Stone-Cech compactification $\beta X$. In some versions of this type of compactification, one embeds $\mathbb{R}$ into the one point compactification $\mathbb{R} \cup\{\infty\}$. We prefer to use the compactification $[-\infty, \infty]$ instead since this space is ordered. Let $g: \mathbb{R} \rightarrow \mathbb{R}$ be a function. We say that $A(X)$ is $g$-invariant if $g \circ f \in A(X)$ for all $f \in A(X)$.

Definition 11. We will say that a vector subspace $A(X)$ of $C(X)$ is adequate if

(a) $A(X)$ separates points from closed sets and contains the constant functions;

(b) There exists a continuous nondecreasing function $g: \mathbb{R} \rightarrow \mathbb{R}$, with $g(t)=0$ if $t \leq 0$ and $g(t)=1$ if $t \geq 1$, such that $A(X)$ is $g$-invariant.

(c) The positive cone $A(X)_{+}$generates $A(X)$, i.e., every $f \in A(X)$ can be written as $f_{1}-f_{2}$, where $f_{1}$ and $f_{2}$ are nonnegative functions in $A(X)$.

Observe that conditions (b) and (c) hold if $A(X)$ is a sublattice of $C(X)$. Indeed, condition (c) is obvious for a sublattice. Take $g(t)$ to be $0, t$ and 1 respectively for $t \leq 0,0<t<1$ and $t \geq 1$ respectively. Then $A(X)$ is $g$-invariant if $A(X)$ is a sublattice of $C(X)$. Also, condition (c) holds if $A(X)$ consists of bounded functions and contains constants. Indeed, in this case, any $f \in A(X)$ can be written as $c 1_{X}-\left(c 1_{X}-f\right)$, where $c \geq 0$ and $c 1_{X} \geq f$. 
Lemma 12. Let $X$ be a completely regular space and let $A(X)$ be an adequate subspace of $C(X)$, Given $x_{0} \in \mathcal{A} X, f \in A(X)$, and a neighborhood $U$ of $x_{0}(f)$ in $[-\infty, \infty]$, set $V=\{x \in \mathcal{A} X: x(f) \in U\}$. Then there exists $h \in A(X)$, with $0 \leq h \leq 1_{X}$ and $x_{0}(h)=0$, such that $W=\{x \in \mathcal{A} X$ : $x(h)<1\} \subseteq V$.

Proof. Let $g$ be the function given in Definition [11. Define $g(\infty)=1$ and $g(-\infty)=0$. Then $x(g \circ f)=g(x(f))$ for all $x \in \mathcal{A} X$ and all $f \in A(X)$. First consider the case where $x_{0}(f)=a \in \mathbb{R}$. Choose $\varepsilon>0$ such that $(a-\varepsilon, a+$ $\varepsilon) \subseteq U$. Set $f_{1}=\varepsilon^{-1}\left(f-a 1_{X}\right) \in A(X)$. Then $x_{0}\left(f_{1}\right)=0$. If $x \in \mathcal{A} X$ and $\left|x\left(f_{1}\right)\right|<1$, then $|x(f)-a|=\varepsilon\left|x\left(f_{1}\right)\right|<\varepsilon$. Thus $W^{\prime}=\left\{x \in \mathcal{A} X:\left|x\left(f_{1}\right)\right|<\right.$ $1\} \subseteq V$. Set $h=1_{X}+g \circ f_{1}-g \circ\left(f_{1}+1_{X}\right) \in A(X)$. Using the observation above, we see that for any $x \in \mathcal{A} X, x(h)=1+g\left(x\left(f_{1}\right)\right)-g\left(x\left(f_{1}\right)+1\right)$. It is easy to check that $0 \leq h \leq 1_{X}, x_{0}(h)=0$, and that $W \subseteq W^{\prime} \subseteq V$.

If $x_{0}(f)=+\infty$ or $-\infty$, the proof is similar. Assume the former. Choose $0<m \in \mathbb{R}$ such that $(m, \infty] \subseteq U$ and define $h=1_{X}-g \circ\left(f-(m+1) 1_{X}\right)$. We omit the verification that $h$ satisfies the requirements.

Denote by $C_{b}(X)$ the subspace of $C(X)$ consisting of the bounded functions. Let $A_{b}(X)=A(X) \cap C_{b}(X)$ and $\hat{A}_{b}(X)=\left\{\hat{f}: f \in A_{b}(X)\right\}$. The map $f \mapsto \hat{f}$ is a bijection from $A_{b}(X)$ onto the subspace $\hat{A}_{b}(X)$ of $C(\mathcal{A} X)$. Since $A_{b}(X)$ contains the constant functions on $X, \hat{A}_{b}(X)$ contains the constant functions on $\mathcal{A} X$.

Lemma 13. Let $X$ be a completely regular space. If $A(X)$ is an adequate subspace of $C(X)$, then $\hat{A}_{b}(X)$ precisely separates points from closed sets in $\mathcal{A} X$.

Proof. Let $x_{0} \in \mathcal{A} X$ and $F$ be a closed subset of $\mathcal{A} X$ not containing $x_{0}$. Then $V=\mathcal{A} X \backslash F$ is an open neighborhood of $x_{0}$. Choose $f_{1}, \ldots, f_{n} \in A(X)$ and open neighborhoods $U_{i}$ of $x_{0}\left(f_{i}\right)$ in $[-\infty, \infty]$ such that $\cap_{i=1}^{n} V_{i} \subseteq V$, where $V_{i}=\left\{x \in \mathcal{A} X: x\left(f_{i}\right) \in U_{i}\right\}$. By Lemma 12, there exist $h_{i}, 1 \leq i \leq n$, such that $0 \leq h_{i} \leq 1_{X}, x_{0}\left(h_{i}\right)=0$, and $W_{i}=\left\{x \in \mathcal{A} X: x\left(h_{i}\right)<1\right\} \subseteq V_{i}$. Set $h=1_{X}-g \circ \sum_{i=1}^{n} h_{i} \in A(X)$. Then $0 \leq h \leq 1_{X}$ and $\hat{h}\left(x_{0}\right)=x_{0}(h)=1$. If $x \in \mathcal{A} X, x \notin V$, then there exists $j$ such that $x \notin V_{j}$, and hence $x \notin W_{j}$. Thus $x\left(h_{j}\right) \geq 1$ and so $x\left(\sum_{i=1}^{n} h_{i}\right) \geq 1$. Therefore, $\hat{h}(x)=x(h)=0$.

Theorem 14. Let $X$ and $Y$ be completely regular spaces. Suppose that $A(X)$ and $A(Y)$ are adequate subspaces of $C(X)$ and $C(Y)$ respectively. If $T: A(X) \rightarrow A(Y)$ is a linear order isomorphism such that $T\left(A_{b}(X)\right)=$ $A_{b}(Y)$, then there exists a homeomorphism $h: \mathcal{A} X \rightarrow \mathcal{A} Y$ such that for all $x \in X, y \in Y, f \in A(X)$ and $g \in A(Y), \hat{f}\left(h^{-1}(y)\right), \hat{g}(h(x)) \in \mathbb{R}, T f=$ $T 1_{X} \cdot \hat{f} \circ h^{-1}{ }_{\mid Y}$ and $T^{-1} g=T^{-1} 1_{Y} \cdot \hat{g} \circ h_{\mid X}$.

Proof. It is clear that $T$ induces a linear order isomorphism $\hat{T}: \hat{A}_{b}(X) \rightarrow$ $\hat{A}_{b}(Y)$ given by $\hat{T} \hat{f}=(T f)^{r}$. The spaces $\hat{A}_{b}(X)$ and $\hat{A}_{b}(Y)$ are subspaces of $C(\mathcal{A} X)$ and $C(\mathcal{A} Y)$ respectively. By Lemma 13, $\hat{A}_{b}(X)$ and $\hat{A}_{b}(Y)$ precisely 
separate points from closed sets. Therefore, by Theorem 1 and the remark following Proposition 6, there is a homeomorphism $h: \mathcal{A} X \rightarrow \mathcal{A} Y$ such that $\hat{T} \hat{f}=\left(T 1_{X}\right)^{\hat{y}} \cdot \hat{f} \circ h^{-1}$ for all $\hat{f} \in \hat{A}_{b}(X)$ and $\hat{T}^{-1} \hat{g}=\left(T^{-1} 1_{Y}\right)^{\wedge} \cdot \hat{g} \circ h$ for all $\hat{g} \in \hat{A}_{b}(Y)$. Since $T^{-1} 1_{Y}$ is bounded, we get in particular that

$$
1_{\mathcal{A} Y}=\hat{T}\left(T^{-1} 1_{Y}\right)^{\wedge}=\left(T 1_{X}\right)^{\wedge} \cdot\left(T^{-1} 1_{Y}\right)^{\wedge} \circ h^{-1} .
$$

Let $f \in A(X)$ with $f \geq 0$. Suppose that $x_{0} \in \mathcal{A} X$ and $y_{0}=h\left(x_{0}\right) \in Y$. Note that $\hat{f}\left(x_{0}\right) \geq 0$. If $\hat{f}\left(x_{0}\right)=0$, then $T f\left(y_{0}\right) \geq\left(T 1_{X}\right)\left(y_{0}\right) \hat{f}\left(x_{0}\right)$. Otherwise, for all $a \in \mathbb{R}$ with $0 \leq a<\hat{f}\left(x_{0}\right)$, there exists an open neighborhood $U$ of $x_{0}$ in $\mathcal{A} X$ such that $f(x)>a$ for all $x \in U \cap X$. By Lemma 13, there exists $g \in A_{b}(X)$ such that range $\hat{g} \subseteq[0,1], \hat{g}\left(x_{0}\right)=1$ and $\hat{g}=0$ outside $U$. Note that $(T g)\left(y_{0}\right)=\left(T 1_{X}\right)\left(y_{0}\right) \hat{g}\left(x_{0}\right)=\left(T 1_{X}\right)\left(y_{0}\right)$ since $g \in A_{b}(X)$ and $y_{0} \in Y$. As $f-a g \geq 0, T(f-a g) \geq 0$. Thus $(T f)\left(y_{0}\right) \geq a(T g)\left(y_{0}\right)=a\left(T 1_{X}\right)\left(y_{0}\right)$. This shows that $(T f)\left(y_{0}\right) \geq\left(T 1_{X}\right)\left(y_{0}\right) \hat{f}\left(x_{0}\right)$. Since $T 1_{X}\left(y_{0}\right)=\left(T 1_{X}\right)^{\prime}\left(y_{0}\right)>$ 0 by Proposition 5 , we see that, in particular, $\hat{f}\left(x_{0}\right) \in \mathbb{R}$. In other words, $\hat{f}\left(h^{-1}(y)\right) \in \mathbb{R}$ and $(T f)(y) \geq\left(T 1_{X}\right)(y) \hat{f}\left(h^{-1}(y)\right)$ for all $y \in Y$. By symmetry and equation (11), we also get that for all $x \in X$,

$$
\begin{aligned}
\left(T 1_{X}\right)^{\gamma}(h(x)) \cdot f(x) & =\left(T 1_{X}\right)^{\gamma}(h(x)) \cdot\left(T^{-1}(T f)\right)(x) \\
& \geq\left(T 1_{X}\right)^{\prime}(h(x)) \cdot\left(T^{-1} 1_{Y}\right)(x) \cdot(T f)^{\wedge}(h(x)) \\
& =(T f)^{\wedge}(h(x)) .
\end{aligned}
$$

Given $y \in Y$, let $\left(x_{\alpha}\right)$ be a net in $X$ so that $\left(h\left(x_{\alpha}\right)\right)$ converges to $y$. Applying the preceding calculation to $x_{\alpha}$ and taking limit gives $T 1_{X}(y) \cdot \hat{f}\left(h^{-1}(y)\right) \geq$ $(T f)(y)$. Thus $(T f)(y)=\left(T 1_{X}\right)(y) \hat{f}\left(h^{-1}(y)\right)$ for all $y \in Y$.

For a general $f \in A(X)$, write $f=f_{1}-f_{2}$, where $0 \leq f_{1}, f_{2} \in A(X)$. If $y \in Y, \hat{f}_{1}\left(h^{-1}(y)\right)$ and $\hat{f}_{2}\left(h^{-1}(y)\right) \in \mathbb{R}$. By the previous paragraph,

$$
\begin{aligned}
T f(y) & =T f_{1}(y)-T f_{2}(y)=T 1_{X}(y) \cdot\left(\hat{f}_{1}\left(h^{-1}(y)\right)-\hat{f}_{2}\left(h^{-1}(y)\right)\right) \\
& =T 1_{X}(y) \cdot \hat{f}\left(h^{-1}(y)\right) .
\end{aligned}
$$

The formula for $T^{-1} g$ follows by the same argument.

Remark. If $T: A(X) \rightarrow A(Y)$ is a linear order isomorphism so that there exists $0<c<1$ so that $c 1_{Y} \leq T 1_{X} \leq c^{-1} 1_{Y}$, then $T\left(A_{b}(X)\right)=A_{b}(Y)$. This holds in particular if $T 1_{X}=1_{Y}$.

Proof. In fact, if $f \in A_{b}(X)$, then there exists $0<M<\infty$ such that $-M 1_{X} \leq f \leq M 1_{X}$. Then

$$
-M c^{-1} 1_{Y} \leq-M T 1_{X} \leq T f \leq M T 1_{X} \leq M c^{-1} 1_{Y} .
$$

Since the condition $c 1_{Y} \leq T 1_{X} \leq c^{-1} 1_{Y}$ is equivalent to $c 1_{X} \leq T^{-1} 1_{Y} \leq$ $c^{-1} 1_{X}$, the other direction follows by symmetry.

Theorem 14 applies to all function spaces that are commonly considered in the context of order isomorphisms. Given a function space $A(X)$, let $A^{\text {loc }}(X)$ be the space of all real-valued functions $f$ on $X$ such that for every 
$x_{0} \in X$, there are a neighborhood $U$ of $x_{0}$ and a function $g \in A(X)$ such that $f=g$ on the set $U$. The space $A_{b}^{\mathrm{loc}}(X)$ is the subspace of all bounded functions in $A^{\text {loc }}(X)$.

Proposition 15. (a) Let $X$ be a completely regular space and let $A(X)$ be a sublattice of $C(X)$ that separates points from closed sets and contains the constant functions. Then $A(X)$ is adequate.

(b) If $A(X)$ is adequate, then so are $A_{b}(X), A^{\mathrm{loc}}(X)$ and $A_{b}^{\mathrm{loc}}(X)$.

(c) Let $X$ be an open set in a Banach space $E$ and let $p \in \mathbb{N} \cup\{\infty\}$. Suppose that $E$ supports a $C^{p}$ bump function, i.e., there exists $\varphi \in$ $C^{p}(E)$ such that $\varphi(0) \neq 0$ and that $\varphi$ has bounded support. Then $C^{p}(X)$ is adequate.

Proof. For part (a), see the remark following Definition 11, Part (b) is clear. For part (c), take $g: \mathbb{R} \rightarrow \mathbb{R}$ to be a nondecreasing $C^{\infty}$ function such that $g(t)=0$ if $t \leq 0$ and $g(t)=1$ if $t \geq 1$. Then $C^{p}(X)$ and $C_{b}^{p}(X)$ are $g$-invariant.

Let $(X, d)$ be a metric space. The space of Lipschitz functions on $X$, $\operatorname{Lip}(X)$, consists of all real-valued functions $f$ on $X$ such that

$$
\sup \left\{\frac{|f(x)-f(y)|}{d(x, y)}: x, y \in X, x \neq y\right\}<\infty .
$$

The space $\operatorname{lip}(X)$ of little Lipschitz functions consists of all $f \in \operatorname{Lip}(X) \operatorname{such}$ that

$$
\lim _{t \rightarrow 0} \sup \left\{\frac{|f(x)-f(y)|}{t}: 0<d(x, y)<t\right\}=0 .
$$

The space of uniformly continuous functions on $X$ is denoted by $U C(X)$. $\operatorname{Lip}(X), \operatorname{lip}(X)$ and $U C(X)$ are sublattices of $C(X)$ that contain the constant functions. $\operatorname{Lip}(X)$ and $U C(X)$ always separate points from closed sets and hence are adequate by Proposition 15. If $\operatorname{lip}(X)$ separates points from closed sets, then it is also adequate. This occurs in particular if there exists $0<\alpha<1$ and a metric $D$ on $X$ so that $d=D^{\alpha}$. By Proposition 15(c), the local, bounded, and bounded local versions of these spaces are also adequate.

An obvious question with regard to Theorem 14 is under what circumstances would the homeomorphism $h$ map $X$ onto $Y$. A simple example shows that this may not always be the case. In fact, for any completely regular space $X, C_{b}(X)$ is linearly order isomorphic to $C(\beta X)$. Thus, if $X$ and $Y$ are non-homeomorphic spaces with homeomorphic Stone-Čech compactifications, then $C_{b}(X)$ is linearly order isomorphic to $C_{b}(Y)$ under a linear isomorphism that preserves bounded functions.

\section{REFinements}

The purpose of this section is to refine Theorem 14 by showing that in many situations, the homeomorphism $h$ maps $X$ onto $Y$. We will also show that in some cases, one may remove the condition that $T$ preserves bounded functions. First we look at a classical situation. A completely regular space 
$X$ is said to be realcompact if given $x \in \beta X \backslash X$, there exists $f \in C(X)$ whose extension $\hat{f}$ takes infinite value at $x$.

Theorem 16. Let $X$ and $Y$ be realcompact spaces and let $T: C(X) \rightarrow$ $C(Y)$ be a linear order isomorphism. Then there exists a homeomorphism $\theta: X \rightarrow Y$ such that $T f=T 1_{X} \cdot f \circ \theta^{-1}$ and $T^{-1} g=T^{-1} 1_{Y} \cdot g \circ \theta$ for all $f \in C(X)$ and all $g \in C(Y)$.

Proof. Let $u=1_{X}+T^{-1} 1_{Y}$. Observe that $u \geq 1_{X}$ and $T u \geq 1_{Y}$. Define $S: C(X) \rightarrow C(Y)$ by $S f=T(u f) / T u$ for all $f \in C(X)$. It is easy to check that $S$ is a linear order isomorphism such that $S 1_{X}=1_{Y}$. Let $h: \beta X \rightarrow \beta Y$ be the homeomorphism obtained by applying Theorem 14 to the map $S$. Since $\hat{f}\left(h^{-1}(y)\right) \mathbb{R}$ for all $f \in C(X)$ and $y \in Y$, and $X$ is realcompact, we find that $h^{-1}(y) \in X$ for all $y \in Y$. Similarly, $h(x) \in Y$ for all $x \in X$. Let $\theta$ be the restriction of $h$ to $X$. Then $\theta$ is a homeomorphism from $X$ onto $Y$. By Theorem 14 again, and $S f=f \circ \theta^{-1}$ for all $f \in C(X)$. In particular, $(1 / u) \circ \theta^{-1}=S\left(\frac{1}{u}\right)=\frac{T 1_{X}}{T u}$. Hence, for all $f \in C(X)$,

$$
\begin{aligned}
T f & =T u \cdot S\left(\frac{f}{u}\right)=T u \cdot \frac{f}{u} \circ \theta^{-1}=T u \cdot\left(f \circ \theta^{-1}\right) \cdot\left(\frac{1}{u} \circ \theta^{-1}\right) \\
& =T u \cdot\left(f \circ \theta^{-1}\right) \cdot \frac{T 1_{X}}{T u}=T 1_{X} \cdot\left(f \circ \theta^{-1}\right) .
\end{aligned}
$$

Similarly, $T^{-1} g=T^{-1} 1_{Y} \cdot g \circ \theta$ for all $g \in C(Y)$.

Representation of nonlinear order isomorphisms between spaces of continuous functions on compact Hausdorff spaces has been obtained by F. Cabello Sánchez 4 .

For the remainder of this section, we consider metric spaces $X$ and $Y$.

Proposition 17. Let $X$ and $Y$ be metric spaces and let $A(X)$ and $A(Y)$ be adequate subspaces of $C(X)$ and $C(Y)$ respectively. If $h: \mathcal{A} X \rightarrow \mathcal{A} Y$ is a homeomorphism and $x_{0} \in X$, then there is sequence $\left(y_{n}\right)$ in $Y$ that converges to $h\left(x_{0}\right)$ in $\mathcal{A} Y$.

Proof. In the first instance, suppose that $x_{0}$ is an isolated point in $X$. Since $A(X)$ separates points from closed sets, the characteristic function $\chi_{\left\{x_{0}\right\}} \in$ $A(X)$. Let $U=\left\{x \in \mathcal{A} X: x\left(\chi_{\left\{x_{0}\right\}}\right)>0\right\}$. Then $U$ is an open neighborhood of $x_{0}$ in $\mathcal{A} X$. We claim that $U$ contains only $x_{0}$. To this end, suppose that $x \in \mathcal{A} X \backslash\left\{x_{0}\right\}$. Choose a net $\left(x_{\alpha}\right)$ in $X$ that converges to $x$. Since $\chi_{\left\{x_{0}\right\}}\left(x_{\alpha}\right) \in\{0,1\}$ and $\lim _{\alpha} \chi_{\left\{x_{0}\right\}}\left(x_{\alpha}\right)=x\left(\chi_{\left\{x_{0}\right\}}\right)$, the latter value is either 0 or 1 . If it is 1 , then there exists $\alpha_{0}$ such that $\chi_{\left\{x_{0}\right\}}\left(x_{\alpha}\right)=1$ for all $\alpha \geq \alpha_{0}$. Then $x_{\alpha}=x_{0}$ for all $\alpha \geq \alpha_{0}$. As a result, $x=x_{0}$, yielding a contradiction. Thus $x\left(\chi_{\left\{x_{0}\right\}}\right)=0$, i.e., $x \notin U$, as claimed. By the claim, $x_{0}$ is an isolated point in $\mathcal{A} X$. Hence $h\left(x_{0}\right)$ is an isolated point in $\mathcal{A} Y$. But since $Y$ is dense in $\mathcal{A} Y, h\left(x_{0}\right)$ cannot be in $\mathcal{A} Y \backslash Y$. So $h\left(x_{0}\right) \in Y$ and the conclusion of the proposition is obvious.

Now suppose that $x_{0}$ is not an isolated point in $X$. Fix a pairwise distinct sequence of points $\left(x_{n}\right)$ in $X$ that converges to $x_{0}$ and a strictly positive null 
sequence $\left(\varepsilon_{n}\right)$ in $\mathbb{R}$. Choose $f_{n} \in A(X)$ such that $f_{n}\left(x_{n}\right)=1$ and $f_{n}=0$ outside $B\left(x_{n}, \varepsilon_{n}\right)$. Set $U_{n}=\left\{x \in \mathcal{A} X: x\left(f_{n}\right)>0\right\}$. Then $U_{n}$ is an open neighborhood of $x_{n}$ in $\mathcal{A} X$. Thus $h\left(U_{n}\right)$ is a nonempty open set in $\mathcal{A} Y$ and hence $h\left(U_{n}\right) \cap Y \neq \emptyset$. Pick $y_{n} \in h\left(U_{n}\right) \cap Y$ and let $z_{n}=h^{-1}\left(y_{n}\right)$. Take any $f \in A(X)$. In particular, $f$ is continuous on $X$. For any $\varepsilon>0$, there exists $\delta>0$ so that $\left|f(x)-f\left(x_{0}\right)\right|<\varepsilon$ if $x \in B\left(x_{0}, \delta\right)$. Observe that $U_{n}$ is open in $\mathcal{A} X$ and hence $U_{n} \subseteq \overline{U_{n} \cap X}$. Then $z_{n} \in U_{n} \subseteq \overline{U_{n} \cap X}$. By continuity of $\hat{f}$, $z_{n}(f)=\hat{f}\left(z_{n}\right) \in \overline{f\left(U_{n} \cap X\right)}$. There exists $n_{0}$ such that $B\left(x_{n}, \varepsilon_{n}\right) \subseteq B\left(x_{0}, \delta\right)$ for all $n \geq n_{0}$. Hence $U_{n} \cap X \subseteq B\left(x_{0}, \delta\right)$ for all $n \geq n_{0}$. Therefore, $\left|z_{n}(f)-f\left(x_{0}\right)\right| \leq \varepsilon$ for all $n \geq n_{0}$. This proves that $\lim z_{n}(f)=f\left(x_{0}\right)$. As $f \in A(X)$ is arbitrary, $z_{n} \rightarrow x_{0}$ in $\mathcal{A} X$. Thus $y_{n}=h\left(z_{n}\right) \rightarrow h\left(x_{0}\right)$ in $\mathcal{A} Y$, as desired.

A set of points $S$ in a metric space is separated if there exists $\varepsilon>0$ such that $d\left(x_{1}, x_{2}\right) \geq \varepsilon$ whenever $x_{1}$ and $x_{2}$ are distinct points in $S$.

Corollary 18. Let $X$ and $Y$ be metric spaces and let $A(X)$ and $A(Y)$ be adequate subspaces of $C(X)$ and $C(Y)$ respectively. Assume that

(a) $A(Y)=A^{\text {loc }}(Y)$ or $A_{b}^{\text {loc }}(Y)$, or

(b) $Y$ is complete and that for any separated sequence $\left(y_{n}\right)$ in $Y$, there exists $g \in A(Y)$ such that $g\left(y_{2 n}\right)=1$ and $g\left(y_{2 n-1}\right)=0$ for all $n$.

If $h: \mathcal{A} X \rightarrow \mathcal{A} Y$ is a homeomorphism and $x_{0} \in X$, then $h\left(x_{0}\right) \in Y$.

Proof. (a) By Proposition 17, there is a sequence $\left(y_{n}\right)$ in $Y$ that converges to $h\left(x_{0}\right)$. If $\left(y_{n}\right)$ has a subsequence that converges in $Y$, then we are done. Assume that $\left(y_{n}\right)$ has no subsequence that converges in $Y$. We may then assume that there is a strictly positive real sequence $\left(\varepsilon_{n}\right)$ so that $B\left(y_{m}, 2 \varepsilon_{m}\right) \cap B\left(y_{n}, 2 \varepsilon_{n}\right)=\emptyset$ if $m \neq n$. By Lemma 13, there exists $g_{n} \in A(Y)$ so that $g_{n}\left(y_{n}\right)=1, g_{n}=0$ outside $B\left(y_{n}, \varepsilon_{n}\right)$, and $0 \leq g_{n} \leq 1_{Y}$. Let $g$ be the pointwise sum $\sum g_{2 n}$. Take $y \in Y$. If $y \in B\left(y_{n}, 2 \varepsilon_{n}\right)$ for some $n$, then there exists $\varepsilon>0$ such that $B(y, \varepsilon)$ does not intersect $B\left(y_{m}, \varepsilon_{m}\right)$ for any $m \neq n$. Suppose $y \notin B\left(y_{n}, 2 \varepsilon_{n}\right)$ for any $n$. If, for all $\varepsilon>0, B(y, \varepsilon)$ intersects $B\left(y_{n}, \varepsilon_{n}\right)$ for at least two $n$, then for any $\varepsilon>0, B(y, \varepsilon)$ intersects infinitely many $B\left(y_{n}, \varepsilon_{n}\right)$. This implies that $\left(y_{n}\right)$ has a convergent subsequence, contrary to the choice of $\left(y_{n}\right)$. This establishes that there exists $\varepsilon>0$ so that $B(y, \varepsilon)$ intersects at most one $B\left(y_{n}, \varepsilon_{n}\right)$. Thus $g$ is a bounded function in $A^{\mathrm{loc}}(Y)$. By the assumption, $g \in A(Y)$. In particular, $\left(g\left(y_{n}\right)\right)$ converges to $\hat{g}\left(h\left(x_{0}\right)\right)$. However, this is impossible since $g\left(y_{n}\right)=1$ if $n$ is even and 0 if $n$ is odd.

(b) By Proposition 17, there is a sequence $\left(y_{n}\right)$ in $Y$ that converges to $h\left(x_{0}\right)$. If $\left(y_{n}\right)$ has a Cauchy subsequence, then we are done. Otherwise, we may assume that $\left(y_{n}\right)$ is separated. By the assumption, there exists $g \in A(Y)$ so that $g\left(y_{2 n}\right)=1$ and $g\left(y_{2 n-1}\right)=0$ for all $n$. But this is impossible since $\left(g\left(y_{n}\right)\right)$ converges to $\hat{g}\left(h\left(x_{0}\right)\right)$. 
The next theorem unifies many results concerning unital order isomorphisms on most types of commonly considered function spaces defined on metric spaces.

Theorem 19. Let $X$ and $Y$ be metric spaces. Assume that $A(X)$ is an adequate subspace of $C(X)$ and that either

(a) $A(X)=A^{\mathrm{loc}}(X)$ or $A_{b}^{\mathrm{loc}}(X)$, or

(b) $X$ is complete and for any separated sequence $\left(x_{n}\right)$ in $X$, there exists $f \in A(X)$ such that $f\left(x_{2 n}\right)=1$ and $f\left(x_{2 n-1}\right)=0$ for all $n$.

Assume the same for $A(Y)$. If $T: A(X) \rightarrow A(Y)$ is a linear order isomorphism such that $T\left(A_{b}(X)\right)=A_{b}(Y)$, then there is a homeomorphism $h: X \rightarrow Y$ such that $T f=T 1_{X} \cdot f \circ h^{-1}$ and $T^{-1} g=T 1_{Y} \cdot g \circ h$ for all $f \in A(X)$ and $g \in A(Y)$.

Proof. Apply Theorem 14 and Corollary 18 (a) or (b) as the case may be.

Theorem [19 applies if $A(X)$ (and $A(Y)$ ) is of one of the following types:

(a) $\operatorname{Lip}(X), \operatorname{Lip}_{b}(X) U C(X), U C_{b}(X)$, where $X$ is complete metric;

(b) $\operatorname{lip}(X), \operatorname{lip}_{b}(X)$, where $X$ is complete metric and $\operatorname{lip}(X)$ satisfies condition (b) in Theorem 19.

(c) $\operatorname{Lip}^{\operatorname{loc}}(X), \operatorname{Lip}_{b}^{\text {loc }}(X), U C^{\text {loc }}(X), U C_{b}^{\text {loc }}(X)$, where $X$ is metric (not necessarily complete);

(d) $\operatorname{lip}^{\text {loc }}(X), \operatorname{lip}_{b}^{\text {loc }}(X)$, where $X$ is metric, not necessarily complete, and $\operatorname{lip}^{\text {loc }}(X)$ separates points from closed sets;

(e) $C^{p}(X), C_{b}^{p}(X)$, where $X$ is an open set in a Banach space $E$ that supports a $C^{p}$ bump function.

Finally, we show that the condition that $T$ preserves bounded functions may be removed in certain cases. The idea is to use the "division trick" that has been employed in the proof of Theorem [16.

Theorem 20. Let $X$ and $Y$ be metric spaces. Assume that

(a) $A(X)$ is a sublattice of $C(X)$ that separates points from closed sets and contains constants, and that either

(i) $A(X)=A^{\mathrm{loc}}(X)$ or $A_{b}^{\mathrm{loc}}(X)$, or

(ii) $X$ is complete and $A(X)=\operatorname{Lip}(X)$ or $\operatorname{Lip}_{b}(X)$; or

(iii) $A(X)=\operatorname{lip}(X)$ or $\operatorname{lip}_{b}(X)$, where $X$ is complete with metric $d$ such that $d=D^{\alpha}$ for some metric $D$ on $X$ and $0<\alpha<1$; or

(b) $A(X)=C^{p}(X)$ or $C_{b}^{p}(X)$, where $X$ is an open set in a Banach space $E$ that supports a $C^{p}$ bump function.

Assume the same for $A(Y)$. If $T: A(X) \rightarrow A(Y)$ is a linear order isomorphism, then there is a homeomorphism $h: X \rightarrow Y$ such that $T f=$ $T 1_{X} \cdot f \circ h^{-1}$ and $T^{-1} g=T 1_{Y} \cdot g \circ h$ for all $f \in A(X)$ and $g \in A(Y)$.

Proof. Let $u=1_{X}+T^{-1} 1_{Y}$. Then $u \in A(X)$ and $u \geq 1_{X}, T u \geq 1_{Y}$. Define

$$
F(X)=\left\{\frac{f}{u}: f \in A(X)\right\} \quad \text { and } \quad F(Y)=\left\{\frac{g}{T u}: g \in A(Y)\right\} \text {. }
$$


If $A(X)$ is a sublattice of $C(X)$ that separates points from closed sets, then $F(X)$ is a sublattice of $C(X)$ that contains constants and separates points from closed sets. If $A(X)=C^{p}(X)$, then $F(X)=C^{p}(X)$. If $A(X)=$ $C_{b}^{p}(X)$, then $u$ is bounded and $\geq 1_{X}$. Hence $F(X)=C_{b}^{p}(X)$. Thus, in all cases, $F(X)$ is an adequate subspace of $C(X)$. The same considerations apply to $F(Y)$. Define a map $S: F(X) \rightarrow F(Y)$ by $S f=T(u f) / T u$ for all $f \in F(X)$. It is easy to check that $S$ is a linear isomorphism such that $S 1_{X}=$ $1_{Y}$. From the remark following Theorem 14 , we see that $S\left(F_{b}(X)\right)=F_{b}(Y)$. Denote the $F(X)$ - and $F(Y)$ - compactifications of $X$ and $Y$ respectively by $\mathcal{F} X$ and $\mathcal{F} Y$ respectively. By Theorem 14, there is a homeomorphism $h: \mathcal{F} X \rightarrow \mathcal{F} Y$ such that $S f=\hat{f} \circ h^{-1}{ }_{\mid Y}$ for all $f \in F(X)$. Thus

$$
T f(y)=T u(y) \cdot(f / u)^{r}\left(h^{-1}(y)\right) \quad \text { for all } f \in A(X) \text { and all } y \in Y \text {. }
$$

If $h$ is a homeomorphism from $X$ onto $Y$, then it follows from (2) that $T 1_{X}(y)=T u(y) / u\left(h^{-1}(y)\right)$ and hence $T f(y)=T 1_{X}(y) \cdot f\left(h^{-1}(y)\right)$. The result for $T^{-1} g$ can be obtained similarly. By symmetry, it remains to show that $h(X) \subseteq Y$.

If $A(Y)=A^{\mathrm{loc}}(Y)$ or $A_{b}^{\mathrm{loc}}(Y)$ (including the cases where $A(Y)=C^{p}(Y)$ or $C_{b}^{p}(Y)$ ), then $F(Y)=F^{\text {loc }}(Y)$ or $F_{b}^{\text {loc }}(Y)$ (note that in the latter case $T u$ is bounded and also bounded away from 0 ). So by Corollary $18, h(X) \subseteq Y$.

Finally, we consider case (a)(ii). For case (a)(iii), a similar argument works using the metric $D$. Suppose that $Y$ is complete and $A(Y)=\operatorname{Lip}(Y)$ or $\operatorname{Lip}_{b}(Y)$. Let $x_{0} \in X$ and $y_{0}=h\left(x_{0}\right)$. By Proposition [17, there exists a sequence $\left(y_{n}\right)$ in $Y$ that converges to $y_{0}$ in $\mathcal{F} Y$. If $\left(y_{n}\right)$ has a Cauchy subsequence in $Y$, then $y_{0} \in Y$. Otherwise, by using a subsequence, we may assume that $\left(y_{n}\right)$ is separated. In the first instance, suppose that $\left(T u\left(y_{n}\right)\right)$ has a bounded subsequence. Then we may assume without loss of generality that $\left(T u\left(y_{n}\right)\right)$ converges to a real number $a$. Applying (2) with $y=y_{n}$ and taking limit, we see that $\lim T f\left(y_{n}\right)=a \cdot f\left(x_{0}\right) / u\left(x_{0}\right) \in \mathbb{R}$ for any $T f \in A(Y)$. But since $\left(y_{n}\right)$ is a separated sequence in $Y$ and $\operatorname{Lip}_{b}(Y) \subseteq$ $A(Y)$, this is not true. Hence it must be that $\left(T u\left(y_{n}\right)\right)$ diverges to $\infty$. By choosing a subsequence if necessary, we may assume that $T u\left(y_{n}\right)>4 T u\left(y_{m}\right)$ if $m<n$. Note that there is a constant $C>0$ such that $d\left(y_{m}, y_{n}\right) \geq$ $C\left|T u\left(y_{m}\right)-T u\left(y_{n}\right)\right|$. Then

$$
d\left(y_{m}, y_{n}\right) \geq \frac{C}{2}\left(T u\left(y_{m}\right)+T u\left(y_{n}\right)\right) \quad \text { if } m<n .
$$

Hence the balls $B\left(y_{n}, \frac{C}{2} T u\left(y_{n}\right)\right)$ are pairwise disjoint. Furthermore, as $T u$ is unbounded, $A(Y)=\operatorname{Lip}(Y)$. In this case, it is easy to construct a function $g$ in $\operatorname{Lip}(Y)$ such that $g\left(y_{2 n-1}\right)=\frac{C}{2} T u\left(y_{2 n-1}\right)$ and $g\left(y_{2 n}\right)=0$. However, it follows from (22) that $\lim T f\left(y_{n}\right) / T u\left(y_{n}\right)=f\left(x_{0}\right) / u\left(x_{0}\right)$ for any $f \in A(X)$. Taking $f=T^{-1} g$ yields a contradiction. 


\section{REFERENCES}

[1] J. Araujo, Separating maps and linear isometries bwteen some spaces of continuous functions, J. Math. Anal. Appl 226 (1998), 23-39.

[2] J. Araujo, Realcompactness and Banach-Stone theorems, Bull. Belg. Math. Soc. Simon Stevin 10(2003), 247-258.

[3] S. Banach, Théorie des Opérations Linéaires, Warszawa 1932. Reprinted, Chelsea Publishing Company, New York, 1963.

[4] F. Cabello SÁnchez, Homomorphisms on lattices of continuous functions, Positivity 12(2008), 341-362.

[5] F. Cabello Sánchez and J. Cabello Sánchez, Some preserver problems on algebras of smooth functions, Ark. Math., 48(2010), 289-300.

[6] F. Cabello Sánchez and J. Cabello Sánchez, Nonlinear isomorphisms of lattices of Lipschitz functions , Houston J. Math., 37(2011), .

[7] I. Gelfand And A. Kolmogorov, On rings of continuous functions on topological spaces, Dokl. Akad. Nauk. SSSR 22 (1939), 11 - 15.

[8] M.I. Garrido And J.A. Jaramillo, A Banach-Stone theorem for uniformly continuous functions, Monatsh. Math. 131 (2000), 189-192.

[9] M.I. Garrido And J.A. Jaramillo, Homomorphisms on function lattices, Monatsh. Math. 141 (2004), 127-46.

[10] L. Gillman and M. Jerison, Rings of Continuous Functions, Van Nostrand, Princeton, 1960.

[11] A. JimÉnez-VArgas and M. Villegas-Vallecillos, Order isomorphisms of littleLipschitz algebras, Houston J. Math., 34(2008), 1185-1195.

[12] A. JimÉnez-VArgas and M. Villegas-VAllecillos, Homomorphisms on realvalued little Lipschitz function spaces, Topology and Appl., 156(2009), 2908-2913.

[13] R.V. KADISOn, A generalized Schwarz inequality and algebraic invariants for operator algebras, Ann. Math. textbf56(1952), $494-503$.

[14] I. Kaplansky, Lattices of continuous functions, Bull. Amer. Math. Soc. 53 (1947), $617-623$.

[15] M.H. Stone, Applications of the theory of Boolean rings to general topology, Trans. Amer. Math. Soc. 41 (1937), $375-481$.

Department of Mathematics, National University of Singapore, Singapore 119076

E-mail address: matlhh@nus.edu.sg

School of Mathematical Sciences and LPMC, NAnkai University, Tianjin, 300071, CHINA

E-mail address: leilee@nankai.edu.cn 